\title{
MicroRNA-133a-3p inhibits cell proliferation, migration and invasion in colorectal cancer by targeting AQP1
}

\author{
BIN KONG, SHIPENG ZHAO, XIANWU KANG and BO WANG \\ Department of Gastrointestinal Surgery, The Third Affiliated Hospital of Hebei \\ Medical University, Shijiazhuang, Hebei 050051, P.R. China
}

Received July 28, 2020; Accepted March 22, 2021

DOI: $10.3892 / \mathrm{ol} .2021 .12910$

\begin{abstract}
Recently, miR-133a-3p has been identified as a marker for human colorectal cancer (CRC) and the association between miR-133a-3p and aquaporin 1 (AQP1) has been described in endothelial cells. However, the regulatory functions of the miR-133a-3p/AQP1 axis remain unclear in CRC. The present study analyzed the expression of miR-133a-3p and AQP1 in CRC tissues $(n=56)$ and cell lines using reverse transcription-quantitative PCR and western blot analysis. The $\chi^{2}$ test was used to assess the associations between miR-133a-3p/AQP1 and clinicopathological features of patients with CRC. Next, the functional role of miR-133a-3p/AQP1 in CRC was evaluated in vitro by performing Cell Counting Kit- 8 and Transwell assays. Moreover, the online software tool TargetScan7.1 was used to predict AQP1 as the target gene of miR-133a-3p, followed by validation using a luciferase reporter assay. The results showed that miR-133a-3p was significantly downregulated, while AQP1 was upregulated in CRC tissues and cell lines compared with corresponding controls. Clinically, it was demonstrated that miR-133a-3p/AQP1 expression was significantly associated with tumor TNM stage $(\mathrm{P}=0.020)$. Functional experiments indicated that miR-133a-3p-overexpression remarkably suppressed, while knockdown promoted, cell proliferation, migration and invasion in CRC cells. Mechanically, AQP1 was identified and validated as a target gene of miR-133a-3p in CRC cells The expression level of AQP1 mRNA was not correlated with miR-133a-3p expression in CRC tissues. Furthermore, AQP1-knockdown induced, while overexpression reversed, the suppressive effects of miR-133a-3p on CRC cells. Taken together, these findings suggested that miR-133a-3p might be a tumor suppressor by suppressing cell proliferation, migration and invasion via targeting AQP1.
\end{abstract}

Correspondence to: Dr Bin Kong, Department of Gastrointestinal Surgery, The Third Affiliated Hospital of Hebei Medical University, 139 Ziqiang Road, Shijiazhuang, Hebei 050051, P.R. China

E-mail: bin_kong0906@126.com

Key words: colorectal cancer, miR-133a-3p, AQP1, proliferation, migration

\section{Introduction}

Colorectal cancer (CRC) is one of most prevalent malignant gastrointestinal tumor types worldwide and is the leading cause of tumor-associated deaths $(1,2)$. The previously identified risk factors for CRC include obesity, smoking, hereditary factors and chronic intestinal inflammation, accompanied with main symptoms, such as weakness, fatigue and unexplained weight loss (3-5). Currently, great progress has been made in the primary therapeutic strategy for patients with $\mathrm{CRC}$, including surgery, chemotherapy and radiotherapy (6). Unfortunately, the long-term survival of patients with CRC remains poor with $<50 \%$, particularly in patients at advance stage, or with local recurrence and distant metastasis $(7,8)$. A major challenge for treatment of advanced metastatic disease is due to insufficient knowledge on the molecular mechanisms underlying the initiation and development of CRC.

MicroRNAs (miRNAs/miRs), composed of 19-25 nucleotides, are a group of short non-coding endogenous RNA molecules that function as negative regulators on gene expression via binding to the 3' untranslated regions (3'UTRs) of target mRNAs $(9,10)$. Accumulating evidence has suggested that miRNAs are extensively involved in tumor development by participating in biological processes, including cell proliferation, apoptosis, migration and invasion, especially in CRC (11-13). miR-133a-3p, a member of the miRNA family, has been recently studied for its tumor suppressive role in various cancer types by targeting different related molecules. For instance, Huang et al (14) reported that overexpression of miR-133a-3p inhibits the proliferation, migration and invasion abilities of gallbladder carcinoma cells through directly targeting recombination signal-binding protein $\mathrm{J} \kappa$. In esophageal squamous cell carcinoma (ESCC), miR-133a-3p inhibits cell propagation, invasion and migration and facilitated apoptosis by targeting collagen type I $\alpha 1$ (15). The similar suppressive effects of miR-133a-3p on cell proliferation and migration were also demonstrated in retinoblastoma (16), gastric cancer (17) and prostate cancer (18). Notably, a recent study by Zhou et al (19) showed that overexpression of miR-133a-3p inhibits cell proliferation with G1 arrest of CRC cells. Moreover, hsa-miR-133a-3p has been identified as selective marker for human colon cancer by extensive screening of miRNA populations (20). However, the clinical significance of miR-133a-3p and its regulatory function on the malignant behaviors in CRC have not been elucidated yet. 
Aquaporin 1 (AQP1), a member of water channel protein family, is responsible for water passive transport quickly across biological membranes (21). Previous studies have described the important roles for AQP1 acting as an oncogene in carcinogenesis and tumor behavior, including glioblastoma multiforme (22), ovarian cancer (23), osteosarcoma (24) and ESCC (25). Notably, the expression of AQP1 is an independent poor prognostic factor for stage II and III CRC $(26,27)$, but the biological function of AQP1 in CRC remains undetermined. Notably, a recent study by Jiang et al (28) illustrated that the myocyte-specific enhancer factor $2 \mathrm{C}$ and miR-133a-3p regulatory circuit could maintain the homeostasis and physiological function of AQP1 in endothelial cells. Nevertheless, whether AQP1 is a target gene of miR-133a-3p in CRC cell functions still unclear.

In the present study, the expression of miR-133a-3p/AQP1 was determined in CRC tissues and the association between miR-133a-3p/AQP1 expression and clinicopathological features of patients with CRC was evaluated. By performing gain-of-function and loss-of-function assays, the effects of miR-133a-3p or AQP1 on CRC cell proliferation, migration and invasion were investigated. Whether miR-133a-3p regulated CRC cell functions via targeting AQP1 was further validated.

\section{Materials and methods}

Clinical tissue samples. A total of 56 paired tumor tissues and matched adjacent normal tissues (at least 5-cm away from tumor margin) taken from left colon side were collected from patients histologically diagnosed as CRC (age range, 28-81 years) by two independent pathologists during surgical resection between March 2018 and October 2019 at the Third Affiliated Hospital of Hebei Medical University (Hebei, China). Before enrollment, all patients signed the informed written consent and were confirmed not to receive any chemotherapy or radiotherapy. The basic clinical features of all patients are summarized in Table I. Collected tissue samples were immediately frozen in liquid nitrogen and kept at $-80^{\circ} \mathrm{C}$ in a refrigerator. The experimental protocols obtained the approval from The Medial Ethics Committee of the Third Affiliated Hospital of Hebei Medical University (Hebei, China).

Cell culture. The four human CRC cell lines (DLD-1, SW1116, SW480 and HCT116) and a normal colon epithelial cell line (FHC) were provided by the Cell Bank of Type Culture Collection of The Chinese Academy of Sciences. Apart from SW1116 cells cultured in Dulbecco's modified Eagle's medium, all the other cell lines were cultured in RPMI-1640 medium (both HyClone; Cyvita). All media were supplemented with $10 \%$ fetal bovine serum (FBS; Gibco; Thermo Fisher Scientific, Inc.) and placed at $37^{\circ} \mathrm{C}$ in a humidified incubator with $5 \% \mathrm{CO}_{2}$.

Cell transfection. For miR-133a-3p-overexpression or AQP1-knockdown, miR-133a-3p mimics (5'-UUUGGUCCCC UUCAACCAGCUG-3'), mimics negative control (NC: 5'-CAG CUGGUUGAAGGGGACCAAA-3'), miR-133a-3p inhibitor (5'-GGGCAATGAAATCCCTGTGAT-3'), inhibitor NC (5'-TTC TCCGAACGTGTCACGTTTC-3'), small interring RNA targeting AQP1 (si-AQP1) and si-NC were chemically synthesized by Guangzhou RiboBio Co.,Ltd. In addition, the coding sequences of AQP1 were synthesized by RiboBio and then cloned into pcDNA3.1 to construct a AQP1 expression vector (pcDNA3.1AQP1). Empty pcDNA3.1 vector was served as a NC. DLD-1, SW480 or HCT116 cells were plated in 6-well plates at a density of $3 \times 10^{5}$ cells per well and cell transfection was performed using Lipofectamine ${ }^{\circledR} 2000$ (Invitrogen; Thermo Fisher Scientific, Inc.) for $48 \mathrm{~h}$ at $37^{\circ} \mathrm{C}$, according to the manufacturer's instructions. The concentration of all miRNAs was $50 \mathrm{nM}$ and the concentration of siRNAs was $30 \mathrm{nM}$. For rescue experiments, the concentration of pcDNA3.1-AQP1 was $10 \mu \mathrm{g}$. After $48 \mathrm{~h}$ transfection, cells were harvested for subsequent experiments.

Reverse transcription-quantitative $(R T-q) P C R$. Total RNA was extracted from tissue samples or cell lines with TRIzol ${ }^{\circledR}$ reagent (Invitrogen; Thermo Fisher Scientific, Inc.) and cDNA was synthesized at $37^{\circ} \mathrm{C}$ for $60 \mathrm{~min}$ and at $98^{\circ} \mathrm{C}$ for $10 \mathrm{~min}$ using the TaqMan microRNA Reverse Transcription kit (Applied Biosystems; Thermo Fisher Scientific, Inc.) or ABScript II cDNA First Strand Synthesis kit (Invitrogen; Thermo Fisher Scientific, Inc.). Quantitative real-time PCR was carried out with TaqMan miRNA Assay Probes (Applied Biosystems; Thermo Fisher Scientific, Inc.) or SYBR Premix Ex Taq ${ }^{\mathrm{TM}}$ Real-Time PCR Kit (Thermo Fisher Scientific, Inc.) on an Applied Biosystems 7500 Sequence Detection system (Applied Biosystems; Thermo Fisher Scientific, Inc.) using the following primer sequences: miR-133a-3p, forward, 5'-UUUGGUCCCC UUCAACCAGCUG-3' and reverse, 5'-UAAACCAAGGUAA AAUGGUCGA-3'; U6, forward, 5'-CGCTTCGGCAGCACA TATAC-3' and reverse, 5'-TTC ACG AAT TTG CGT GTC AT-3'; AQP1, forward, 5'-ACCTGCTGGCCATTGACTAC-3' and reverse, 5'-CCAGGGCACTCCCAATGAAT-3'; $\beta$-actin, forward, 5'-CTGTGTGGATTGGTGGCTCT-3' and reverse, 5'-CAGCTCAGTAACAGTCCGCC-3'. The thermocycling conditions were as follows: Pre-degeneration at $95^{\circ} \mathrm{C}$ for $3 \mathrm{~min}$ and 40 cycles of $95^{\circ} \mathrm{C}$ for $30 \mathrm{sec}$, annealing and elongation at $60^{\circ} \mathrm{C}$ for $1 \mathrm{~min}$. All of the reactions were run in triplicate. Relative expression of miR-133a-3p and AQP1 normalized to U6 and $\beta$-actin, respectively, and was calculated using the $2^{-\Delta \Delta \mathrm{Cq}}$ method (29). All experiments were biologically repeated three times.

Cell proliferation assay. Transfected cells at a density of $4 \times 10^{3}$ cells per well were seeded into 96 -well plates and incubated for $0,24,48$ or $72 \mathrm{~h}$. At each time point, cells in each well were incubated with $10 \mu$ l Cell Counting Kit-8 (CCK-8) solution (Beyotime Institute of Biotechnology) according to the manufacturer's instruction. After incubation for another $2 \mathrm{~h}$ at $37^{\circ} \mathrm{C}$, the absorbance at each time point was measured at a wavelength of $450 \mathrm{~nm}$ using a microplate reader. All experiments were biologically repeated three times.

Transwell assay. Cell migration and invasion were assessed with Transwell chambers (Corning, Inc.) precoated with

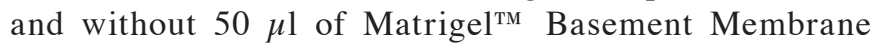
Matrix (BD Biosciences) for $2 \mathrm{~h}$ at $37^{\circ} \mathrm{C}$, respectively. For the Transwell assay, transfected DLD-1, SW480 or HCT116 cells were suspended in FBS-free culture medium and added to the upper chamber $\left(2 \times 10^{4}\right.$ cells/well). Meanwhile, $500 \mu 1$ of medium containing 20\% FBS (Gibco; Thermo Fisher Scientific, Inc.) was added to the lower chamber. After $24 \mathrm{~h}$ incubation at $37^{\circ} \mathrm{C}$, the cells that migrated to the lower 
Table I. Association between miR-133a-3p expression and clinicopathological characteristics in 56 patients with colorectal cancer.

\begin{tabular}{|c|c|c|c|c|}
\hline \multirow[b]{2}{*}{ Characteristics } & \multirow[b]{2}{*}{$\begin{array}{c}\text { Value, } \\
\mathrm{n}\end{array}$} & \multicolumn{2}{|c|}{$\begin{array}{l}\text { miR-133a-3p } \\
\text { expression }\end{array}$} & \multirow[b]{2}{*}{ P-value } \\
\hline & & $\begin{array}{c}\text { Low } \\
(n=31)\end{array}$ & $\begin{array}{l}\text { High } \\
(n=25)\end{array}$ & \\
\hline Age, years & & & & 0.877 \\
\hline$<60$ & 24 & 13 & 11 & \\
\hline$\geq 60$ & 32 & 18 & 14 & \\
\hline Sex & & & & 0.489 \\
\hline Male & 33 & 17 & 16 & \\
\hline Female & 23 & 14 & 9 & \\
\hline Tumor size, cm & & & & 0.968 \\
\hline$<5$ & 36 & 20 & 16 & \\
\hline$\geq 5$ & 20 & 11 & 9 & \\
\hline Stage & & & & $0.020^{\mathrm{a}}$ \\
\hline $\mathrm{I} / \mathrm{II}$ & 32 & 22 & 10 & \\
\hline III/IV & 24 & 9 & 15 & \\
\hline Differentiation & & & & 0.453 \\
\hline Well/moderate & 26 & 13 & 13 & \\
\hline Poor & 30 & 18 & 12 & \\
\hline
\end{tabular}

${ }^{\mathrm{a}} \mathrm{P}<0.05$. miR, microRNA.

chamber were fixed with $10 \%$ methanol for $30 \mathrm{sec}$ at $37{ }^{\circ} \mathrm{C}$ and stained with $0.1 \%$ crystal violet in methanol for $15 \mathrm{~min}$ at room temperature. The migratory or invasive cells were counted in randomly selected five fields of view under an a light microscope (Olympus Corporation; magnification, x100). All experiments were biologically repeated three times.

Luciferase reporter assay. The sites of miR-133a-3p binding with AQP1 gene were predicted using the online software tool TargetScan7.1 (http://www.targetscan. org). For the luciferase reporter assay, the AQP1 3'UTR wild-type (WT) or mutant (MUT) was inserted into the pmirGLO luciferase reporter vector (Promega Corporation), named as pmirGLO-AQP1 3'UTR-WT or pmirGLO-AQP1 3'UTR-MUT, respectively. The pmirGLO-AQP1 3'UTR-WT contained the predicted miR-133a-3p binding sites, whereas pmirGLO-AQP1 3'UTR-MUT was constructed using the site-directed mutagenesis kit (Takara Bio, Inc.) to encompass a mutated miR-133a-3p binding site. Next, DLD-1 or SW480 cells were co-transfected with miR-133a-3p mimics or mimics NC together with AQP1 WT or AQP1 MUT, respectively, using Lipofectamine ${ }^{\circledR}$ 2000. Subsequently, the luciferase activities of Firefly and Renilla were measured using Dual Luciferase Assay System (Promega Corporation) after 48 h of transfection. Relative luciferase activity was calculated as the ratio of Firefly luciferase activity vs. Renilla luciferase activity.

Western blot analysis. Total protein sample was extracted from cell lines with RIPA lysis buffer and protein concentration was determined using the BCA protein assay kit (both from Beyotime Institute of Biotechnology). Then, equal amount of protein sample $(30 \mu \mathrm{g})$ was separated on 10\% SDS-PAGE and transferred onto PVDF membranes (EMD Millipore). Blocking of membranes was performed with $5 \%(\mathrm{w} / \mathrm{v})$ skimmed milk in Tris buffered saline with $0.2 \%$ Tween-20 (TBST) for $2 \mathrm{~h}$ at room temperature. Then, the membranes were incubated with primary antibodies against AQP1 (1:1,000; cat. no. ab168387; Abcam) and GAPDH (1:5,000; cat. no. ab8245; Abcam) overnight at $4^{\circ} \mathrm{C}$. After washed with TBST, membranes were incubated with goat anti-rabbit IgG-horseradish peroxidase secondary antibody (MBS435036; MyBioSource) for $2 \mathrm{~h}$ at room temperature and visualized via an enhanced chemiluminescence detection system (Santa Cruz Biotechnology, Inc.).

Statistical analysis. All experiments were performed in triplicate and data are presented as mean \pm standard deviation. Statistical analysis was performed using GraphPad Prism version 6.0 software (GraphPad Software, Inc.). All patients with CRC were classified into low-expression group and high-expression group using the median value $(0.385$ for miR-133a-3p and 2.54 for AQP1) as the cut-off. The $\chi^{2}$ test was used to assess the associations between miR-133a-3p or AQP1 and clinicopathological features of patients with CRC. Spearman's correlation analysis was conducted to assess the correlation between miR-133a-3p and AQP1. Two groups were compared using unpaired Student's t-test or one-way ANOVA, followed by Tukey's post hoc test, was used for more than two groups. $\mathrm{P}<0.05$ was considered to indicate a statistically significant difference.

\section{Results}

Downregulation of $\mathrm{miR}-133 a-3 p$ in $C R C$ is associated with TNM stage. The expression of miR-133a-3p was determined in paired tumor and matched adjacent normal tissues in a cohort of 56 patients with CRC. The results from RT-qPCR analysis showed that miR-133a-3p expression was significantly downregulated in CRC tissues compared with matched normal tissues (Fig. 1A). Similarly, miR-133a-3p expression within CRC cell lines (DLD-1, SW1116, SW480 and HCT116) was remarkably increased in comparison to the normal colon epithelial cell line FHC (Fig. 1B). By analyzing the association between miR-133a-3p expression and clinicopathological parameters, it was reported that miR-133a-3p expression was significantly associated with tumor stage $(\mathrm{P}=0.020)$, but not associated with age, sex, tumor size and differentiation (Table I).

miR-133a-3p suppresses the proliferation, migration and invasion of CRC cells. Synthesized miR-133a-3p mimics or mimics NC were transfected into DLD-1 and SW480 cells with relatively lower endogenous miR-133a-3p expression. As shown in Fig. 2A, RT-qPCR analysis verified that miR-133a-3p expression was significantly increased in DLD-1 and SW480 cells after miR-133a-3p mimics transfection compared with mimics $\mathrm{NC}$ transfection. Next, the biological function of miR-133a-3p on these two transfected CRC cell lines was investigated. The CCK- 8 assay indicated that overexpression of miR-133a-3p significantly inhibited the proliferation rate in both DLD-1 and 

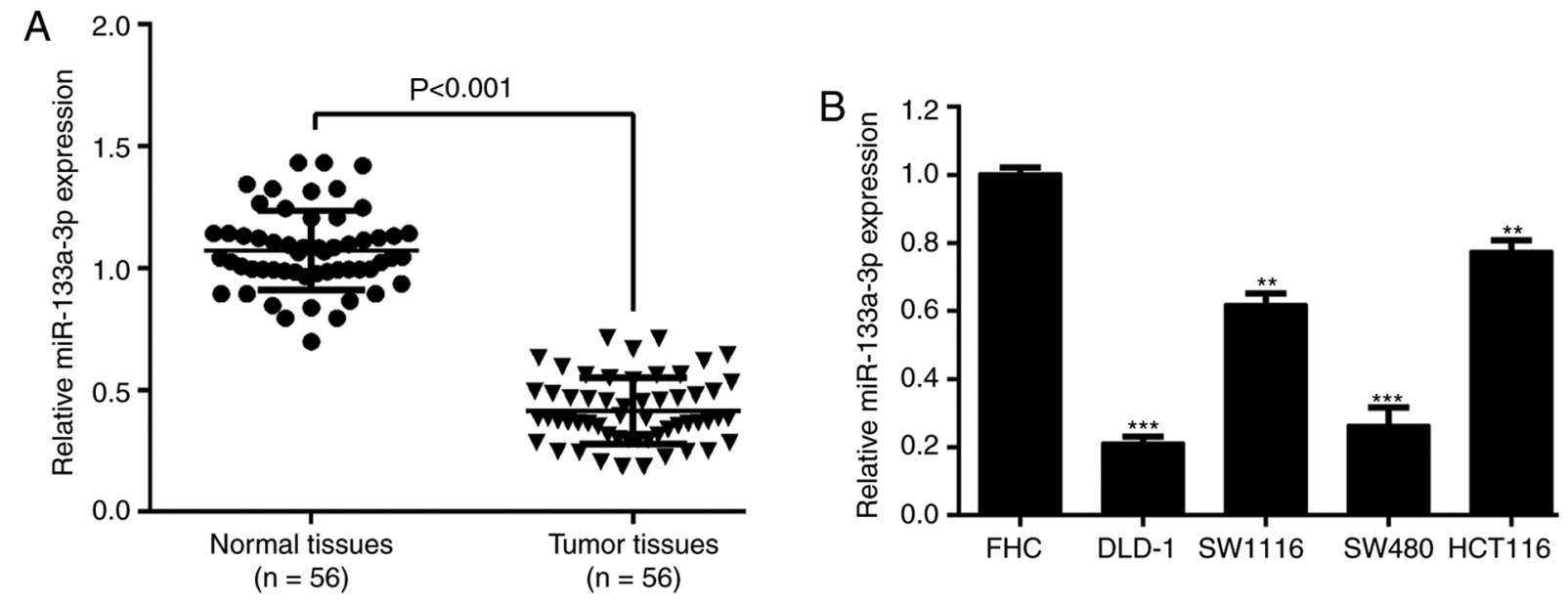

Figure 1. miR-133a-3p is downregulated in CRC tissues and cell lines. Reverse transcription-quantitative PCR results determined miR-133a-3p levels in (A) tumor and matched adjacent normal tissues of patients with CRC ( $\mathrm{n}=56$ ), as well as (B) four human CRC cell lines (DLD-1, SW1116, SW480 and HCT116) and a normal colon epithelial cell line (FHC). ${ }^{* *} \mathrm{P}<0.01$ and ${ }^{* * * *} \mathrm{P}<0.001$ compared with FHC cells. miR, microRNA; CRC, colorectal cancer.

SW480 cells (Fig. 2B). In addition, the effects of miR-133a-3p mimics on cell migration and invasion were also examined using a Transwell assay. As expected, the number of migratory cells was notably reduced after miR-133a-3p mimics transfection in DLD-1 and SW480 cells (Fig. 2C). Consistently, miR-133a-3p-overexpression suppressed the invasive ability of DLD-1 and SW480 cells (Fig. 2D). Furthermore, a loss-of-function assay was performed in HCT116 cells with relatively higher endogenous miR-133a-3 compared with the other three CRC cell lines to further confirm the suppressive effects of miR-133a-3p in CRC cells. As shown in Fig. 2E, miR-133a-3p expression was significantly decreased in HCT116 cells after miR-133a-3p inhibitor transfection compared with inhibitor $\mathrm{NC}$ transfection. The functional assay further demonstrated that knockdown of miR-133a-3p significantly promoted cell proliferation (Fig. 2F), migration and invasion (Fig. 2G) ability in HCT116 cells.

miR-133a-3p directly decreases AQP1 expression by binding to its 3 'UTR. To explore the functional targets of miR-133a-3p in CRC, online software tool TargetScan7.1 predicted that the 3'UTR of AQP1 contains miR-133a-3p binding sites (Fig. 3A). Next, AQP1 3'UTR-WT or AQP1 3'UTR-MUT was transfected with miR-133a-3p mimics or mimics NC into DLD-1 and SW480 cells. The luciferase reporter assay showed that the luciferase activities of AQP1-WT-transfected DLD-1 (Fig. 3B) or SW480 (Fig. 3C) cells significantly decreased upon miR-133a-3p-overexpression. However, the inhibitory effects were abolished when the putative miR-133a-3p seed binding regions in the AQP1 3'UTR were mutated. Moreover, the effect of miR-133a-3p on AQP1 expression was analyzed. As shown in Fig. 3D, the protein expression of AQP1 was downregulated after miR-133a-3p mimics transfection in DLD-1 and SW620 cells. The results demonstrated that miR-133a-3p can negatively regulate $\mathrm{AQP} 1$ expression by interacting with its 3'UTR, which supported AQP1 mRNA as a putative target of miR-133a-3p. In addition, the expression of AQP1 mRNA in paired tumor and matched adjacent normal tissues was determined in a cohort of 56 patients with CRC. As depicted in Fig. 3E, the AQP1 mRNA level was significantly upregu-
Table II. Association between AQP1 expression and clinicopathological characteristics in 56 patients with colorectal cancer.

\begin{tabular}{|c|c|c|c|c|}
\hline \multirow[b]{2}{*}{ Characteristics } & \multirow[b]{2}{*}{ Cases } & \multicolumn{2}{|c|}{ AQP1 expression } & \multirow[b]{2}{*}{ P-value } \\
\hline & & $\begin{array}{l}\text { High } \\
(\mathrm{n}=29)\end{array}$ & $\begin{array}{c}\text { Low } \\
(\mathrm{n}=27)\end{array}$ & \\
\hline Age, years & & & & 0.189 \\
\hline$<60$ & 24 & 10 & 14 & \\
\hline$\geq 60$ & 32 & 19 & 13 & \\
\hline Sex & & & & 0.256 \\
\hline Male & 33 & 15 & 18 & \\
\hline Female & 23 & 14 & 9 & \\
\hline Tumor size, $\mathrm{cm}$ & & & & 0.842 \\
\hline$<5$ & 36 & 19 & 17 & \\
\hline$\geq 5$ & 20 & 10 & 10 & \\
\hline Stage & & & & $0.010^{\mathrm{a}}$ \\
\hline $\mathrm{I} / \mathrm{II}$ & 32 & 22 & 12 & \\
\hline III/IV & 24 & 7 & 17 & \\
\hline Differentiation & & & & 0.058 \\
\hline Well/moderate & 26 & 17 & 9 & \\
\hline Poor & 30 & 12 & 18 & \\
\hline
\end{tabular}

${ }^{\mathrm{a}}<0.05$. AQP1, aquaporin 1.

lated in CRC tissues compared with matched normal tissues. Using the median value of AQP1 mRNA as the cut-off, the results from $\chi^{2}$ test showed that increased AQP1 mRNA expression level was significantly associated with tumor stage ( $\mathrm{P}=0.010$; Table II). However, the expression level of AQP1 mRNA was not correlated with miR-133a-3p expression in CRC tissues (Fig. 3F).

$A Q P 1$ promotes $C R C$ cell proliferation, migration and invasion. To investigate the functional role of AQP1 in CRC in 

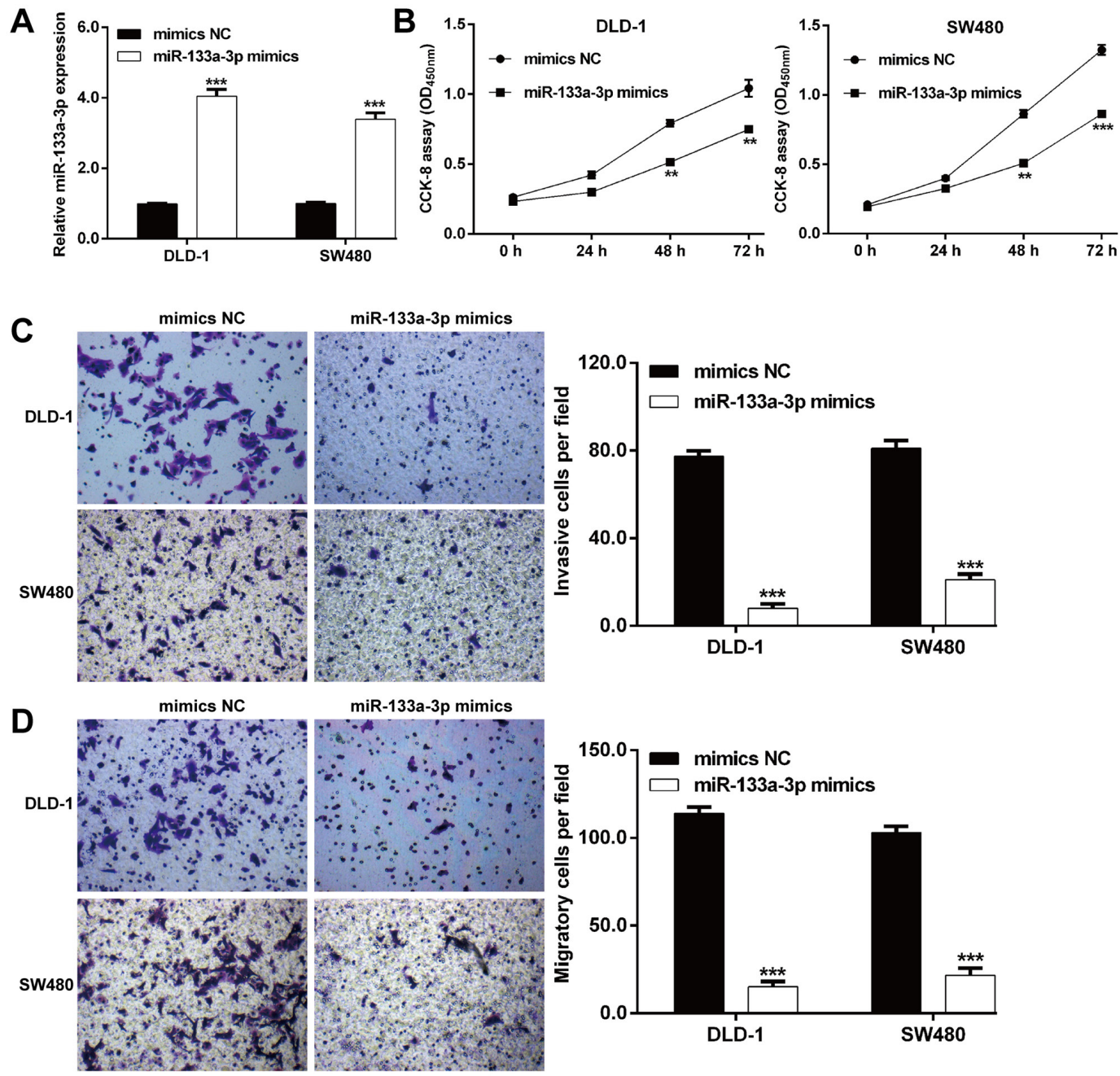

miR-133a-3p mimics

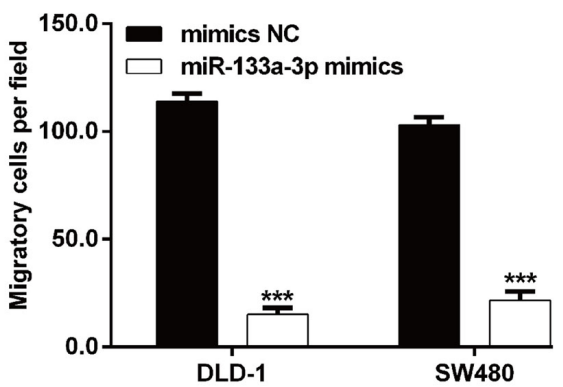

E
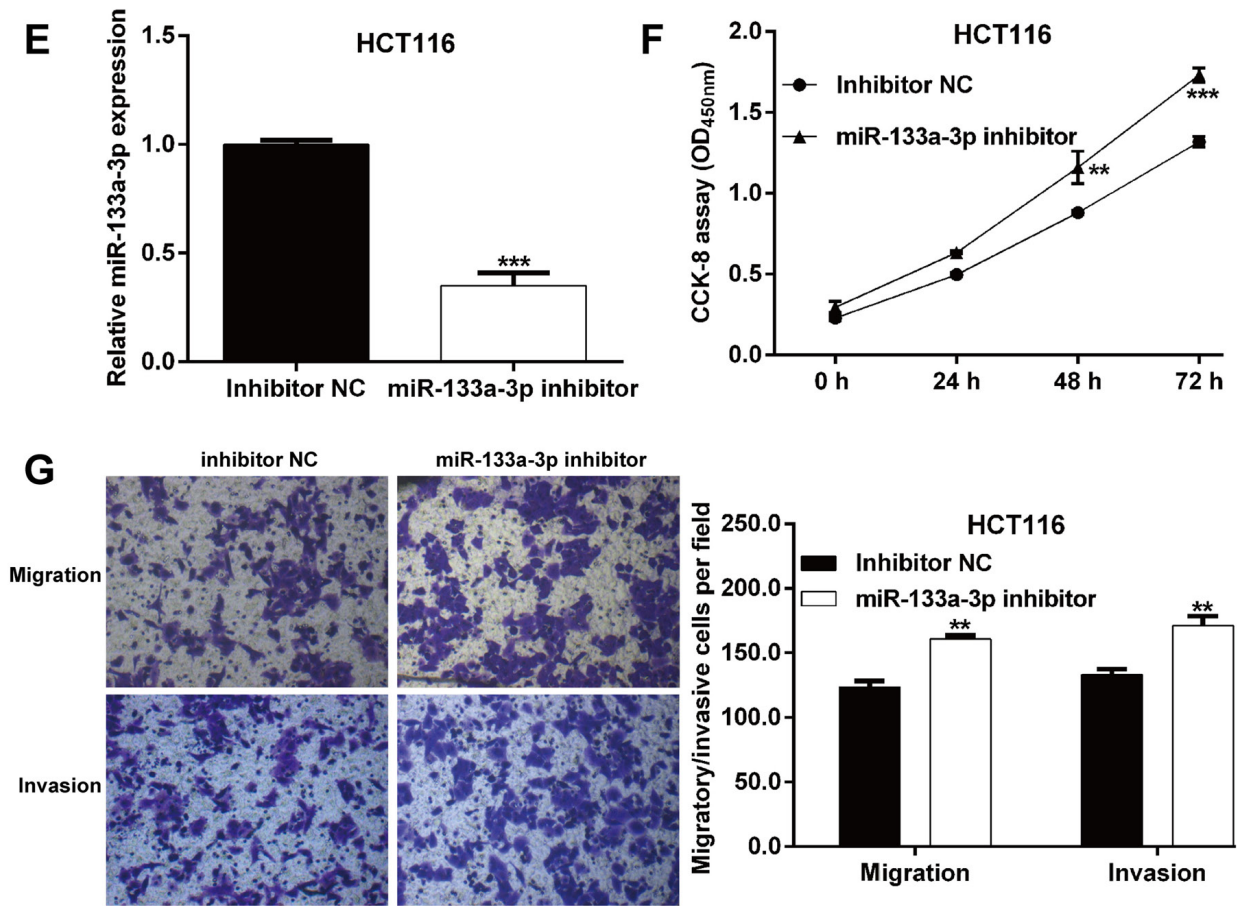

Figure 2. miR-133a-3p suppresses the proliferation, migration and invasion of CRC cells. DLD-1 and SW480 cells were transfected with miR-133a-3p mimics or mimics NC for $48 \mathrm{~h}$. (A) RT-qPCR results showing the transfection efficiency of miR-133a-3p in transfected DLD-1 and SW480 cells. (B) CCK-8 assay tested the effects of miR-133a-3p mimics on cell proliferation of transfected DLD-1 and SW480 cells. Transwell assay was performed to assess (C) cell migration and (D) invasion in transfected DLD-1 and SW480 cells. HCT116 cells were transfected with miR-133a-3p inhibitor or inhibitor NC for 48 h. (E) RT-qPCR results showing the transfection efficiency of miR-133a-3p in transfected HCT116 cells. (F) CCK-8 assay tested the effects of miR-133a-3p inhibitor on cell proliferation of transfected HCT116 cells. (G) A Transwell assay was performed to assess cell migration and invasion in transfected HCT116 cells. ${ }^{* *} \mathrm{P}<0.01$ and ${ }^{* * *} \mathrm{P}<0.001$ compared with respective NC. RT-q, reverse transcription-quantitative; miR, microRNA; NC, negative control; CCK-8, Cell Counting Kit-8. 
A

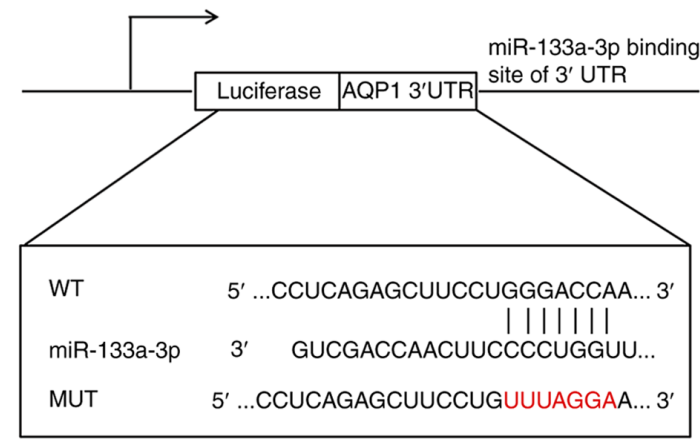

C

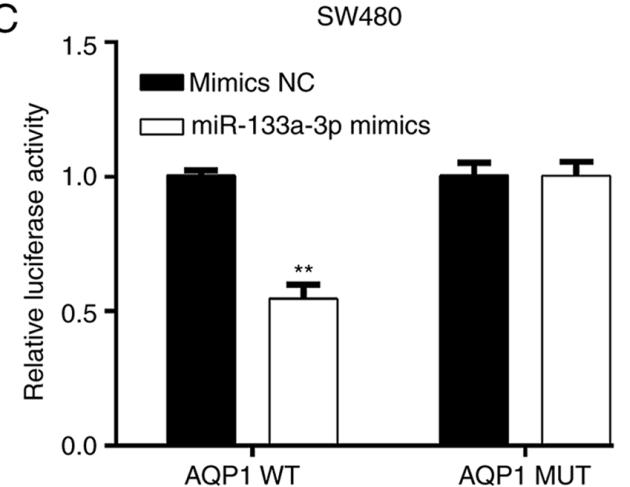

E

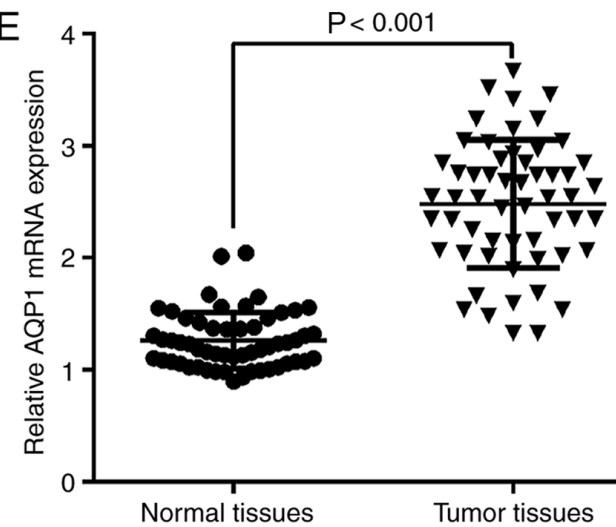

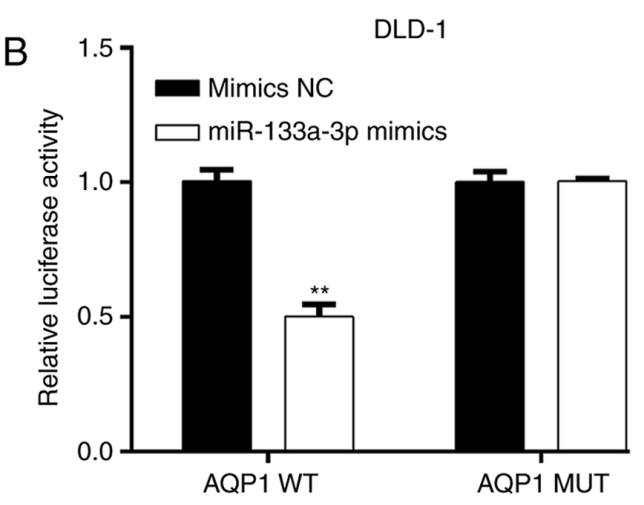
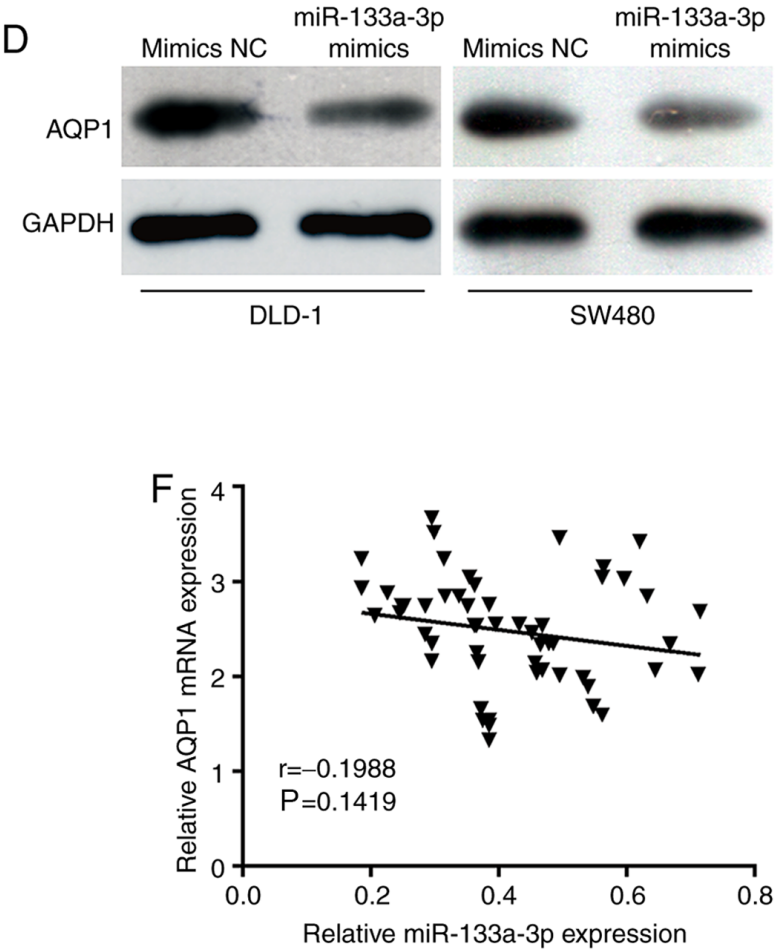

Figure 3. miR-133a-3p regulates AQP1 by binding to its 3'UTR. (A) Putative miR-133a-3p binding sites in the AQP1 3'UTR were revealed using TargetScan. Dual luciferase reporter assay confirmed the binding between miR-133a-3p and AQP1 in (B) DLD-1 and (C) SW480 cells. (D) Western blot assay of AQP1 protein levels in DLD-1 and SW480 cells transfected with miR-133a-3p mimics or mimics NC. (E) Reverse transcription-quantitative PCR results show AQP1 mRNA levels in tumor and matched adjacent normal tissues of patients with CRC $(n=56)$. (F) Spearman's correlation analysis was applied to investigate the correlation between the expressions of miR-133a-3p and AQP1 mRNA in CRC tissues. ${ }^{* *} \mathrm{P}<0.01$ compared with mimics NC. UTR, untranslated region; AQP1, aquaporin 1; WT, wild-type; MUT, mutant; miR, microRNA; NC, negative control; CRC, colorectal cancer; miR, microRNA.

vitro, the expression level of $\mathrm{AQP1}$ protein in four CRC cell lines was determined. As shown in Fig. 4A, the protein level of AQP1 was upregulated in CRC cell lines (DLD-1, SW1116, SW480 and HCT116) compared with the normal colon epithelial cell line FHC. Subsequently, si-AQP1 was transfected into DLD-1 and SW480 cells to perform loss-of-function assays. As depicted in Fig. 4B, the protein expression of AQP1 was downregulated after si-AQP1 transfection in DLD-1 and SW480 cells compared with si-NC transfection. Similar with miR-133a-3p-overexpression, knockdown of AQP1 significantly impaired cell proliferation (Fig. 4C and D), migration (Fig. 4E) and invasion (Fig. 4F) in DLD-1 and SW480 cells. Moreover, the protein level of AQP1 was overexpressed in HCT116 cells following transfection with pcDNA3.1-AQP1, as demonstrated by western blot analysis (Fig. 4G). Contrary to AQP1-knockdown, AQP1-overexpression markedly promoted cell proliferation (Fig. 4H), migration and invasion (Fig. 4I) in HCT116 cells. These data indicated that AQP1 is the target of miR-133a-3p and played a role in CRC cell proliferation, migration and invasion.

Restoration of AQP1 reverses the suppressive effects of miR-133a-3p on CRC cell proliferation, migration and invasion. Furthermore, rescue experiments were performed to investigate whether AQP1 was the downstream functional regulator involved in miR-133a-3p-mediated CRC cell functions. DLD-1 and SW480 cells were co-transfected with miR-133a-3p mimics and pcDNA3.1-AQP1 or empty 

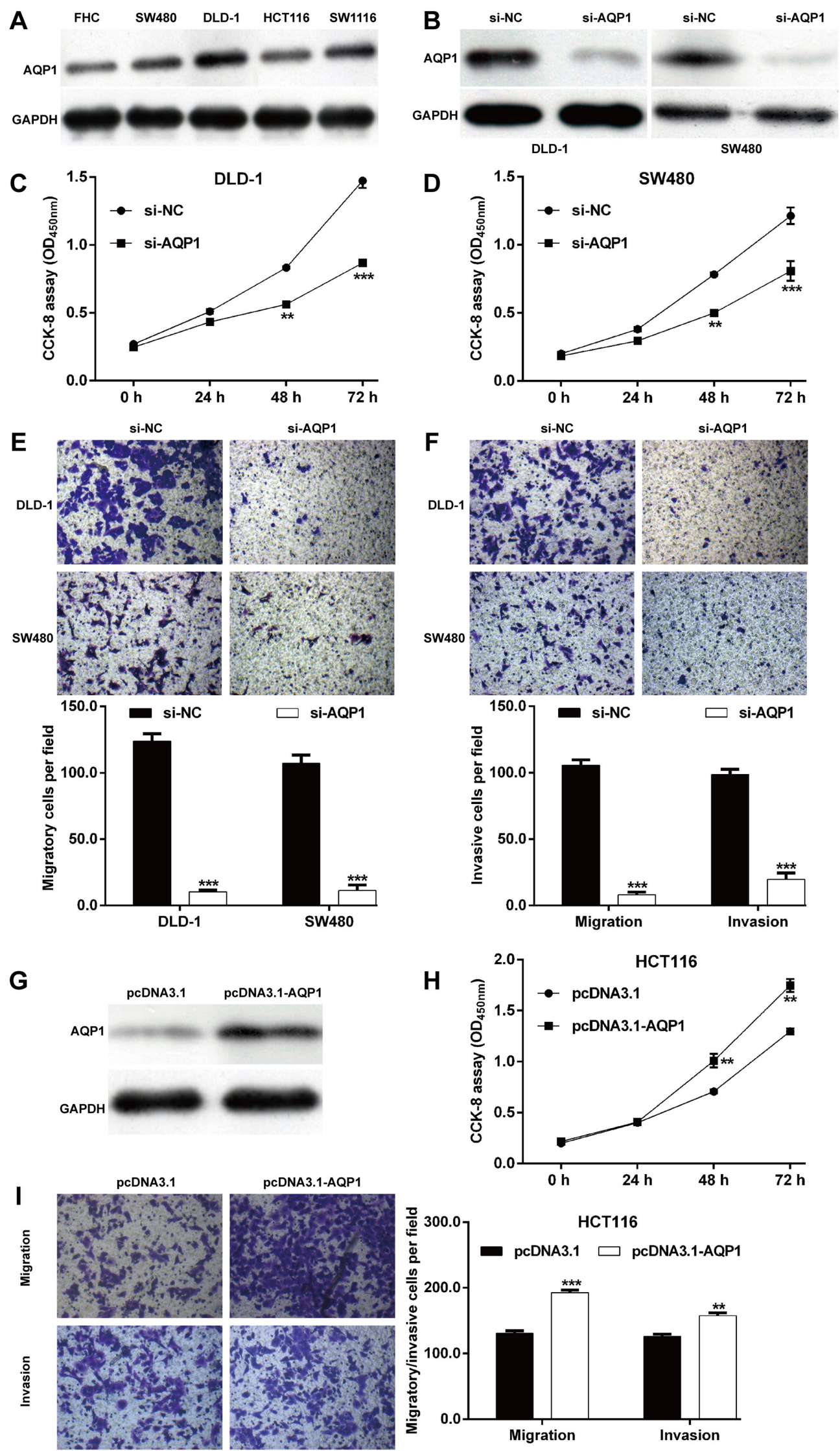

Figure 4. AQP1 promotes CRC cell proliferation, migration and invasion. (A) Expression of AQP1 protein was detected in four human CRC cell lines (DLD-1, SW1116, SW480 and HCT116) and a normal colon epithelial cell line (FHC). DLD-1 and SW480 cells were transfected with si-AQP1 or si-NC for 48 h. (B) Expression of AQP1 protein was detected in transfected DLD-1 and SW480 cells using western blot analysis. (C and D) Proliferation of transfected DLD-1 and SW480 cells was assessed by CCK-8 assay. Transwell assay was performed to assess (E) cell migration and (F) invasion in transfected DLD-1 and SW480 cells. (G) Expression of AQP1 protein was detected in HCT116 cells transfected with pcDNA3.1-AQP1 or pcDNA3.1 using western blot analysis. (H) Proliferation of transfected HCT116 cells was assessed using a CCK-8 assay. (I) Transwell assay was performed to assess cell migration and invasion in transfected HCT116 cells. ${ }^{* * *} \mathrm{P}<0.01$ and ${ }^{* * * *} \mathrm{P}<0.001$ compared with si-NC or pcDNA3.1. AQP1, aquaporin 1; CCK-8, Cell Counting Kit-8; NC, negative control; si, small interfering. 

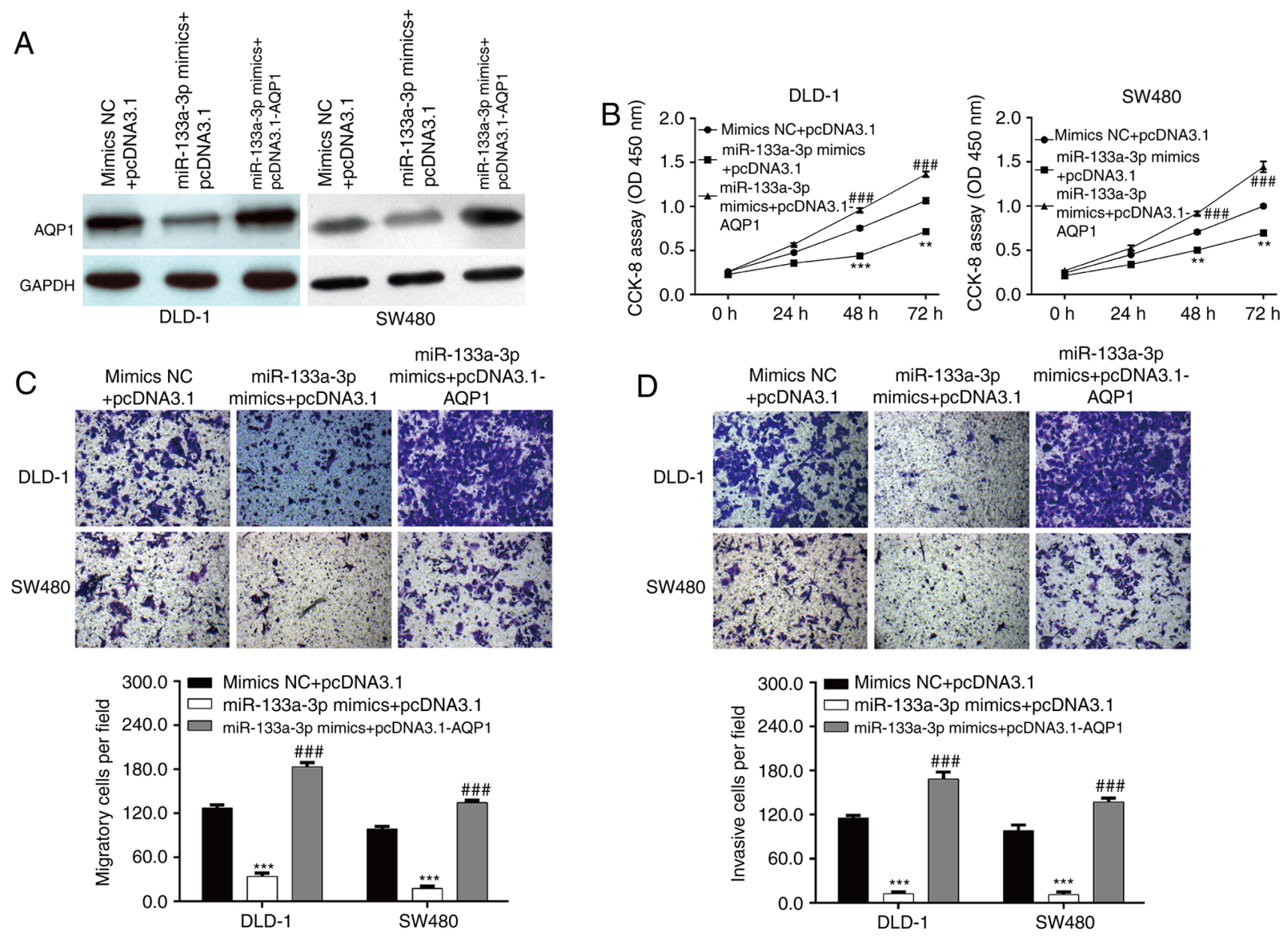

Figure 5. Restoration of AQP1 reverses the suppressive effects of miR-133a-3p on CRC cell proliferation, migration and invasion. DLD-1 and SW480 cells were co-transfected with miR-133a-3p mimics and pcDNA3.1-AQP1 or empty pcDNA3.1 for 48 h. (A) Expression of AQP1 protein was detected in transfected DLD-1 and SW480 cells using western blot analysis. (B) Proliferation of transfected DLD-1 and SW480 cells was assessed using a CCK-8 assay. (C) Transwell assay was performed to assess cell migration and invasion in transfected DLD-1 and SW480 cells. ${ }^{* *} \mathrm{P}<0.01$ and ${ }^{* * *} \mathrm{P}<0.001$ compared with mimics NC + pcDNA3.1.

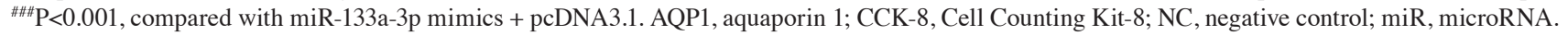

pcDNA3.1. Western blot analysis first confirmed that decreased AQP1 expression induced by miR-133a-3p-overexpression was reversed by pcDNA3.1-AQP1 transfection (Fig. 5A). The in vitro functional experiments, including CCK- 8 and Transwell assays, consistently demonstrated that overexpression of AQP1 significantly abolished the suppressive effects of miR-133a-3p-overexpression on cell proliferation (Fig. 5B), migration (Fig. 5C) and invasion (Fig. 5D) in DLD-1 and SW480 cells. These data suggested that miR-133a-3p negatively regulated CRC cell proliferation, migration and invasion via targeting AQP1.

\section{Discussion}

The present study demonstrated that miR-133a-3p expression was downregulated in CRC tissues compared with adjacent normal tissues. Moreover, decreased miR-133a-3p expression was associated with tumor stage. Similarly, the expression of miR-133a-3p was reduced and linked with clinicopathological parameters of non-small cell lung cancer (30), hepatocellular carcinoma (31) and prostate cancer (18). In addition, miR-133a-3p has been found to be significantly downregulated in human papillomavirus-infected oropharyn- geal squamous cell carcinoma (32), bladder cancer (33), breast cancer (34) and oral squamous cell carcinoma (OSCC) (35). Consistent with the current data, hsa-miR-133a-3p has been identified as selective marker for human colon cancer by extensive screening of miRNA populations (20). These data suggested that miR-133a-3p might be a tumor suppressor in CRC.

Further functional experiments showed that miR-133a-3p exerted suppressive effects on CRC cell proliferation, migration and invasion. As demonstrated by Zhou et al (19), miR-133a-3p is downregulated in CRC tissues and its overexpression inhibits cell proliferation and induces G1/S arrest in CRC cells. Different from this study, the current data not only highlighted the decreased miR-133a-3p expression in CRC tissues, but also indicated its association with the tumor stage of patients with CRC. The in vitro data not only showed the suppressive role of miR-133a-3p on cell proliferation, but also manifested its suppressive effects on cell migration and invasion in CRC cells. On the other hand, addition of miR-133a-3p reduces cell viability, and increases apoptosis and cell cycle arrest in retinoblastoma (16). miR-133a-3p-overexpression could block the activation of autophagy to ruin the abnormal glutaminolysis and further inhibit the proliferation and migra- 
tion/invasion of gastric cancer cells (17). Overexpression of miR-133a-3p suppresses the proliferation, invasion and mitosis of OSCC cells (35). Upregulating miR-133a-3p inhibits cancer stem cell-like phenotypes in vitro and in vivo, as well as attenuates anoikis resistance in vitro in prostate cancer cells (18). In gallbladder carcinoma, Huang et al (14) demonstrated the inhibitory effects of miR-133a-3p on the proliferation, migration and invasion in vitro. These previous studies demonstrate the suppressive effects of miR-133a-3p on the proliferation and malignant behavior of CRC cells.

A previous study by Zhou et al (19) demonstrated that miR-133a-3p suppresses cell proliferation with G1 arrest of CRC cells by targeting SUMO-specific protease 1 expression. Yu et al (36) revealed that miR-133a-3p targets RhoA, which is involved in cytoskeletal reorganization that drives cell motility in CXCL12/CXCR4-induced CRC progression. The present study validated that AQP1 was another target of $\mathrm{miR}-133 \mathrm{a}-3 \mathrm{p}$ and negatively regulated by miR-133a-3p in CRC cells. Clinical analysis showed that increased AQP1 mRNA expression level was associated with tumor stage. The in vitro data suggested that miR-133a-3p exerted its suppressive role in CRC cells might via targeting AQP1. In fact, AQP expression is increased in CRC tissues using immunohistochemical staining (37). AQP1 was identified as a promising candidate as a prognostic biomarker for CRC at TNM stage II and III (26,27). Kang et al (38) found a significant correlation between AQP1 expression and lymph node metastasis in patients with surgically resected colon cancer. Functionally, forced overexpression of AQP1 has been shown to increase angiogenesis, invasion and metastasis in pre-clinical studies of colon adenocarcinoma (39). In the other tumors, including ovarian cancer (23), osteosarcoma (24), breast cancer (40) and melanoma (41), knockdown of AQP1 inhibits cell proliferation and invasion. Notably, the association between miR-133a-3p and AQP1 has also been described in endothelial cells (28). Based on these previous studies, it was thus speculated that miR-133a-3p expression also influences AQP1 not only in non-malignant endothelial cells but also in CRC. Importantly, AQP1 was involved in miR-133a-3p-mediated regulation of $\mathrm{CRC}$ cell functions, functioning as an oncogene. Meanwhile, there were some limitations to the present study, including the lack of in vivo validation for the function of miR-133a-3p/AQP1 axis. Additional targets of miR-133a-3p and the correlation between them should been explored, as well as the use of additional clinical samples to investigate the role of the miR-133a-3p/AQP1 axis in the prognosis of patients with CRC.

In summary, low miR-133a-3p expression levels and high AQP1 expression levels were associated with tumor stage. The current data further showed that miR-133a-3p-overexpression suppressed CRC cell proliferation, migration and invasion, which might be associated with suppression of AQP1 induced by miR-133a-3p. These results may improve our understanding of the role of miR-133a-3p in AQP1-induced proliferation, migration and invasion of $\mathrm{CRC}$, which provides potential therapeutic targets for CRC treatment.

\section{Acknowledgements}

Not applicable.

\section{Funding}

No funding was received.

\section{Availability of data and materials}

All data generated or analyzed during this study are included in this published article.

\section{Authors' contributions}

BK designed this research. SZ and XK carried out most experiments in this work and drafted this manuscript. BW helped with the western blot experiments and help perform statistical analysis. BK and SZ confirmed the authenticity of all raw data. All authors have read and approved the final manuscript.

\section{Ethics approval and consent to participate}

The present study was approved by The Ethics Committee of the Third Affiliated Hospital of Hebei Medical University (Shijiazhuang, China; approval no. G2021-019-1) and performed in accordance with the Declaration of Helsinki.

\section{Patient consent for publication}

Not applicable.

\section{Competing interests}

The authors declare that they have no competing interests.

\section{References}

1. Cai Z and Liu Q: Understanding the Global Cancer Statistics 2018: Implications for cancer control. Sci China Life Sci 64: 1017-1020, 2021.

2. Siegel R, Desantis C and Jemal A: Colorectal cancer statistics, 2014. CA Cancer J Clin 64: 104-117, 2014.

3. Andrews L: Dietary flavonoids for the prevention of colorectal cancer. Clin J Oncol Nurs 17: 671-672, 2013.

4. Altobelli E, Lattanzi A, Paduano R, Varassi G and di Orio F: Colorectal cancer prevention in Europe: Burden of disease and status of screening programs. Prev Med 62: 132-141, 2014.

5. Sugarbaker PH: Colorectal cancer: Prevention and management of metastatic disease. BioMed Res Int 2014: 782890, 2014.

6. Goldstein DA, Zeichner SB, Bartnik CM, Neustadter E and Flowers CR: Metastatic colorectal cancer: A systematic review of the value of current therapies. Clin Colorectal Cancer 15: 1-6, 2016.

7. O'Shannessy DJ, Somers EB, Chandrasekaran LK, Nicolaides NC, Bordeaux J and Gustavson MD: Influence of tumor microenvironment on prognosis in colorectal cancer: Tissue architecture-dependent signature of endosialin (TEM-1) and associated proteins. Oncotarget 5: 3983-3995, 2014.

8. Rawla P, Sunkara T and Barsouk A: Epidemiology of colorectal cancer: Incidence, mortality, survival, and risk factors. Prz Gastroenterol 14: 89-103, 2019.

9. Bartel DP: MicroRNAs: Genomics, biogenesis, mechanism, and function. Cell 116: 281-297, 2004.

10. He L and Hannon GJ: MicroRNAs: Small RNAs with a big role in gene regulation. Nat Rev Genet 5: 522-531, 2004.

11. Guo L, Fu J, Sun S, Zhu M, Zhang L, Niu H, Chen Z, Zhang Y, Guo L and Wang S: MicroRNA-143-3p inhibits colorectal cancer metastases by targeting ITGA6 and ASAP3. Cancer Sci 110: 805-816, 2019.

12. Liu Y, Zhang Y, Wu H, Li Y, Zhang Y, Liu M, Li X and Tang H: miR-10a suppresses colorectal cancer metastasis by modulating the epithelial-to-mesenchymal transition and anoikis. Cell Death Dis 8: e2739, 2017. 
13. Luo F, Zhou J, Wang S, Sun Z, Han Q and Bai C: microRNA-222 promotes colorectal cancer cell migration and invasion by targeting MST3. FEBS Open Bio 9: 901-913, 2019.

14. Huang Y, Wu Y, Dong J, Han D, Yang S and Jiang L: MicroRNA-133a-3p exerts inhibitory effects on gallbladder carcinoma via targeting RBPJ. Am J Cancer Res 6: 2448-2462, 2016.

15. Yin Y, Du L, Li X, Zhang X and Gao Y: miR-133a-3p suppresses cell proliferation, migration, and invasion and promotes apoptosis in esophageal squamous cell carcinoma. J Cell Physiol 234: 12757-12770, 2019.

16. Li J, Liu X, Wang W and Li C: miR-133a-3p promotes apoptosis and induces cell cycle arrest by targeting CREB1 in retinoblastoma. Arch Med Sci 16: 941-956, 2019.

17. Zhang X, Li Z, Xuan Z, Xu P, Wang W, Chen Z, Wang S, Sun G, $\mathrm{Xu} \mathrm{J}$ and $\mathrm{Xu} \mathrm{Z}$ : Novel role of miR-133a-3p in repressing gastric cancer growth and metastasis via blocking autophagy-mediated glutaminolysis. J Exp Clin Cancer Res 37: 320, 2018.

18. Tang Y, Pan J, Huang S, Peng X, Zou X, Luo Y, Ren D, Zhang X, Li R, He P, et al: Downregulation of miR-133a-3p promotes prostate cancer bone metastasis via activating PI3K/AKT signaling. J Exp Clin Cancer Res 37: 160, 2018.

19. Zhou GQ, Han F, Shi ZL, Yu L, Li XF, Yu C, Shen CL, Wan DW, Zhu XG, Li R, et al: miR-133a-3p targets SUMO-specific protease 1 to inhibit cell proliferation and cell cycle progress in colorectal cancer. Oncol Res 26: 795-800, 2018.

20. Weber D, Amar L, Gödde D and Prinz C: Extensive screening of microRNA populations identifies hsa-miR-375 and hsa-miR-133a-3p as selective markers for human rectal and colon cancer. Oncotarget 9: 27256-27267, 2018

21. Verkman AS: More than just water channels: Unexpected cellular roles of aquaporins. J Cell Sci 118: 3225-3232, 2005.

22. Yang WY, Tan ZF, Dong DW, Ding Y, Meng H, Zhao Y, Xin XF and $\mathrm{Bi} \mathrm{W}$ : Association of aquaporin 1 with tumor migration, invasion and vasculogenic mimicry in glioblastoma multiforme. Mol Med Rep 17: 3206-3211, 2018.

23. Wang Y, Fan Y, Zheng C and Zhang X: Knockdown of AQP1 inhibits growth and invasion of human ovarian cancer cells. Mol Med Rep 16: 5499-5504, 2017.

24. Wu Z, Li S, Liu J, Shi Y, Wang J, Chen D, Luo L, Qian Y, Huang X and Wang H: RNAi-mediated silencing of AQP1 expression inhibited the proliferation, invasion and tumorigenesis of osteosarcoma cells. Cancer Biol Ther 16: 1332-1340, 2015.

25. Yamazato Y, Shiozaki A, Ichikawa D, Kosuga T, Shoda K, Arita T, Konishi H, Komatsu S, Kubota T, Fujiwara H, et al: Aquaporin 1 suppresses apoptosis and affects prognosis in esophageal squamous cell carcinoma. Oncotarget 9: 29957-29974, 2018.

26. Yoshida T, Hojo S, Sekine S, Sawada S, Okumura T, Nagata T, Shimada $\mathrm{Y}$ and Tsukada K: Expression of aquaporin-1 is a poor prognostic factor for stage II and III colon cancer. Mol Clin Oncol 1: 953-958, 2013

27. Imaizumi $\mathrm{H}$, Ishibashi $\mathrm{K}$, Takenoshita $\mathrm{S}$ and Ishida $\mathrm{H}$ : Aquaporin 1 expression is associated with response to adjuvant chemotherapy in stage II and III colorectal cancer. Oncol Lett 15: 6450-6456, 2018.

28. Jiang Y, Ma R, Zhao Y, Li GJ, Wang AK, Lin WL, Lan XM, Zhong SY and Cai JH: MEF2C/miR-133a-3p.1 circuit-stabilized AQP1 expression maintains endothelial water homeostasis. FEBS Lett 593: 2566-2573, 2019.
29. Livak KJ and Schmittgen TD: Analysis of relative gene expression data using real-time quantitative PCR and the 2(-Delta Delta C(T)) method. Methods 25: 402-408, 2001

30. Yang ZQ, Wu CA and Cheng YX: Prognostic value of microRNA-133a expression and its clinicopathologic significance in non-small cell lung cancer: A comprehensive study based on meta-analysis and the TCGA database. Oncol Res Treat 41: 762-768, 2018

31. Liang HW, Yang X, Wen DY, Gao L, Zhang XY, Ye ZH, Luo J, $\mathrm{Li} Z \mathrm{Z}, \mathrm{He} \mathrm{Y}$, Pang YY, et al: Utility of miR $133 \mathrm{a} 3 \mathrm{p}$ as a diagnostic indicator for hepatocellular carcinoma: An investigation combined with GEO, TCGA, meta analysis and bioinformatics. Mol Med Rep 17: 1469-1484, 2018.

32. House R, Majumder M, Janakiraman H, Ogretmen B, Kato M, Erkul E, Hill E, Atkinson C, Barth J, Day TA, et al: Smoking-induced control of miR-133a-3p alters the expression of EGFR and HuR in HPV-infected oropharyngeal cancer. PLoS One 13: e0205077, 2018

33. Gao L, Li SH, Tian YX, Zhu QQ, Chen G, Pang YY and Hu XH: Role of downregulated miR-133a-3p expression in bladder cancer: A bioinformatics study. OncoTargets Ther 10: 3667-3683, 2017.

34. Shi W, Tang T, Li X, Deng S, Li R, Wang Y, Wang Y, Xia T, Zhang Y, Zen K, et al: Methylation-mediated silencing of miR-133a-3p promotes breast cancer cell migration and stemness via miR-133a-3p/MAML1/DNMT3A positive feedback loop. J Exp Clin Cancer Res 38: 429, 2019.

35. He B, Lin X, Tian F, Yu W and Qiao B: MiR-133a-3p inhibits oral squamous cell carcinoma (OSCC) proliferation and invasion by suppressing COL1A1. J Cell Biochem 119: 338-346, 2018

36. Yu X, Wang D, Wang X, Sun S, Zhang Y, Wang S, Miao R, Xu X and Qu X: CXCL12/CXCR4 promotes inflammation-driven colorectal cancer progression through activation of RhoA signaling by sponging miR-133a-3p. J Exp Clin Cancer Res 38: $32,2019$.

37. Pei HP, Liu Z, Huang LS and Zhu H: Significance of aquaporin-1 and aquaporin-3 expression in colorectal carcinoma. Zhonghua Wei Chang Wai Ke Za Zhi 14: 275-278, 2011 (In Chinese).

38. Kang BW, Kim JG, Lee SJ, Chae YS, Jeong JY, Yoon GS, Park SY, Kim HJ, Park JS, Choi GS, et al: Expression of aquaporin-1, aquaporin-3, and aquaporin-5 correlates with nodal metastasis in colon cancer. Oncology 88: 369-376, 2015.

39. Dorward HS, Du A, Bruhn MA, Wrin J, Pei JV, Evdokiou A, Price TJ, Yool AJ and Hardingham JE: Pharmacological blockade of aquaporin-1 water channel by AqB013 restricts migration and invasiveness of colon cancer cells and prevents endothelial tube formation in vitro. J Exp Clin Cancer Res 35: 36, 2016.

40. Esteva-Font C, Jin BJ and Verkman AS: Aquaporin-1 gene deletion reduces breast tumor growth and lung metastasis in tumor-producing MMTV-PyVT mice. FASEB J 28: 1446-1453, 2014.

41. Simone L, Gargano CD, Pisani F, Cibelli A, Mola MG, Frigeri A, Svelto $M$ and Nicchia GP: Aquaporin-1 inhibition reduces metastatic formation in a mouse model of melanoma. J Cell Mol Med 22: 904-912, 2018

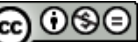

This work is licensed under a Creative Commons Attribution-NonCommercial-NoDerivatives 4.0 International (CC BY-NC-ND 4.0) License. 